\title{
Intra-Symbol Windowing for Egress Reduction in DMT Transmitters
}

\author{
Gert Cuypers, ${ }^{1}$ Koen Vanbleu, $^{2}$ Geert Ysebaert, $^{3}$ and Marc Moonen ${ }^{1}$ \\ ${ }^{1}$ ESAT/SCD-SISTA, Katholieke Universiteit Leuven, 3001 Heverlee, Belgium \\ ${ }^{2}$ Broadcom Corporation, 2800 Mechelen, Belgium \\ ${ }^{3}$ Alcatel Bell, 2018 Antwerp, Belgium
}

Received 28 December 2004; Revised 20 July 2005; Accepted 22 July 2005

\begin{abstract}
Discrete multitone (DMT) uses an inverse discrete Fourier transform (IDFT) to modulate data on the carriers. The high sidelobes of the IDFT filter bank used can lead to spurious emissions (egress) in unauthorized frequency bands. Applying a window function within the DMT symbol can alleviate this. However, window functions either require additional redundancy or will introduce distortions that are generally not easy to compensate for. In this paper, a special class of window functions is constructed that corresponds to a precoding at the transmitter. These do not require any additional redundancy and need only a modest amount of additional processing at the receiver. The results can be used to increase the spectral containment of DMT-based wired communications such as ADSL and VDSL (i.e., asymmetric, resp., very-high-bitrate digital subscriber loop).
\end{abstract}

Copyright (C) 2006 Gert Cuypers et al. This is an open access article distributed under the Creative Commons Attribution License, which permits unrestricted use, distribution, and reproduction in any medium, provided the original work is properly cited.

\section{INTRODUCTION}

Discrete Fourier transform (DFT-) based modulation techniques [1] have become increasingly popular for high-speed communications systems. In the wireless context, for example, for the digital transmission of audio and video, this is usually referred to as orthogonal frequency-division multiplexing (OFDM). Its wired counterpart has been dubbed discrete multitone (DMT), and is employed, for example, for digital subscriber loop (DSL) systems, such as asymmetric DSL (ADSL) and very-high-bitrate DSL (VDSL).

A high bandwidth efficiency is achieved by dividing the available bandwidth into small frequency bands centered around carriers (tones). These carriers are individually modulated in the frequency domain, using the inverse DFT (IDFT). A cyclic prefix (CP) is added to the resulting block of time-domain samples by copying the last few samples and putting them in front of the symbol [2]. This extended block is parallel-to-serialized, passed to a digital-to-analog (DA) convertor and then transmitted over the channel. At the receiver, the signal is sampled and serial-to-parallelized again. The part corresponding to the $\mathrm{CP}$ is discarded, and the remainder is demodulated using the DFT.

In case the order of the channel impulse response does not exceed the $\mathrm{CP}$ length by more than one, equalization can be done easily using a one-tap frequency-domain equalizer
(FEQ) for each tone, correcting the phase shift and attenuation at each tone individually. When the channel impulse response is longer than the $\mathrm{CP}$, the transmission suffers from intercarrier interference (ICI) and intersymbol interference (ISI), requiring more complex receivers, for example, a pertone equalizer (PTEQ) [3]. The windowing technique presented in this article is irrespective of the equalization technique used but can be combined with the PTEQ in a very elegant way.

In addition to a CP, VDSL systems can also use a cyclic suffix (CS). The difference between the CP and CS is irrelevant to this article, therefore they will be treated as one (larger) CP. More importantly, the presence of the CP influences the spectrum of the transmit signal, as will be shown later.

While DMT seems attractive because of its flexibility towards spectrum control, the high sidelobe levels associated with the DFT filter bank form a serious impediment, resulting in an energy transfer between in-band and outof-band signals. This contributes to the crosstalk, for example, between different pairs in a binder, especially for next-generation DSL systems using dynamic spectrum management (DSM), where the transmit band is variable [4]. Moreover, because the twisted pair acts as an antenna [5], there exists a coupling with air signals. The narrowband signals from, for example, an AM broadcast station can 


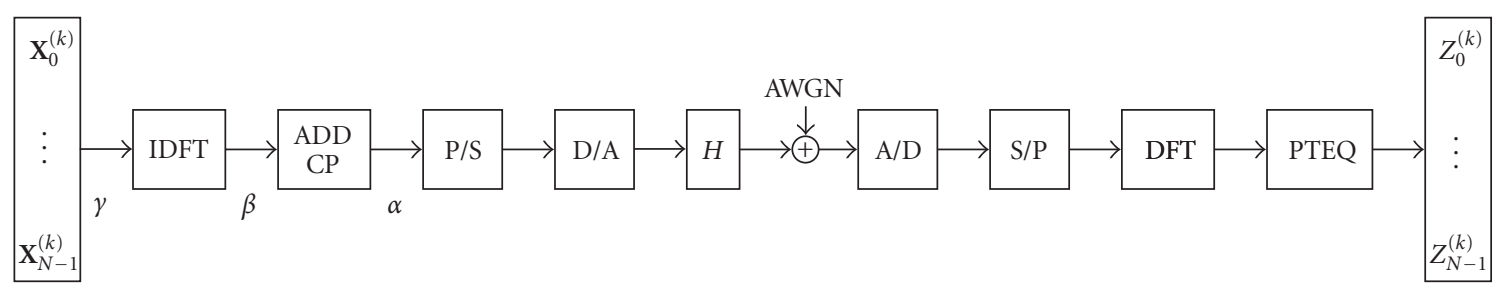

FIgURE 1: Basic DMT system (refer to text for $\alpha$ to $\gamma$ ).

be picked up by the receiver and, due to the sidelobes, be smeared out over a broad frequency tone range. This problem has been recognized, and various schemes have been developed to tackle it (see [6-8]). On the other hand, the same poor spectral containment of transmitted signals makes it difficult to meet egress norms, for example, the ITU-norm [9] specifies that the transmit power of VDSL should be lowered by $20 \mathrm{~dB}$ in the amateur radio bands. Controlling egress is usually done in the frequency domain by combining neighbouring IDFT-inputs (such as in [10]) or, equivalently, by abandoning the DFT altogether and reverting to other filter banks, such as, for example, in [11].

Another approach would be to apply an appropriate time-domain window (see [12] for an overview) at the transmitter. Unfortunately, the application of nonrectangular windows destroys the orthogonality between the tones, resulting in ICI. In [13], a windowed VDSL system is proposed, where the window is applied to additional cyclic continuations of the DMT symbol to prevent distorting the symbol itself.

The technique proposed in this article avoids the overhead resulting from such additional symbol extension by applying the window directly to the DMT symbol, that is, without adding additional guard bands. This windowing is observed to correspond to a precoding operation at the transmitter. Obviously, this alters the frequency content at each carrier, such that a correction at the receiver is needed. While this compensation is generally nontrivial [14], we construct a class of windows that can be compensated for with only a minor amount of additional computations at the receiver.

When investigating transmit windowing techniques, it is important to have an accurate description of the transmit spectrum of DMT/OFDM signals. Although DMT and OFDM are commonplace, a lot of misconception and confusion seem to exist with regard to the nature of their transmit signal spectrum. When working on sampled channel data, the continuous-time character of the line signals is transparent, and therefore usually neglected. However, it is important to realize that the behaviour in between the sample points can be of great importance [15]. The analog signal will generally exceed the sampled points' reach, possibly leading to unnoticed clipping, and hence out-of-band radiation.

Therefore, Section 2 starts by describing the spectrum of the classical DMT signal. The novel windowing system is then presented in Section 3. Section 4 covers the simulation results. Finally, in Section 5, conclusions are presented.

\section{DMT TRANSMIT SIGNAL SPECTRUM}

Consider the DMT system of Figure 1, with DFT-size $N$ and a CP length $\nu$, resulting in a symbol length $L=N+\nu$. The symbol index is $k$ and $\mathbf{X}^{(k)}=\left[X_{0}^{(k)} \cdots X_{N-1}^{(k)}\right]^{T}$ holds the complex subsymbols at tones $i, i=0: N-1$. In a baseband system, such as ADSL, the time-domain signal is realvalued, requiring that $X_{i}^{(k)}=X_{N-i}^{(k) *}$. The corresponding discrete time-domain sample vector (at point $\alpha$ in Figure 1 ) is equal to

$$
\begin{gathered}
\mathbf{x}^{(k)}=\left[x^{(k)}[0], \ldots, x^{(k)}[L-1]\right]^{T}, \\
x^{(k)}[n]=\frac{1}{\sqrt{N}} \sum_{i=0}^{N-1} X_{i}^{(k)} e^{j(2 \pi i / N)(n-v)}, \quad n=0, \ldots, L-1 .
\end{gathered}
$$

Note that the CP is automatically present, due to the periodicity of the complex exponentials. The total discrete timedomain sample stream $x[n]$ is obtained as a concatenation of the individual symbols $\mathbf{x}^{(k)}$. Interpolation of these samples yields the continuous time-domain signal $s(t)$, given by

$$
\begin{gathered}
s(t)=\int_{\tau=-\infty}^{\infty} v(\tau-t)\left[\sum_{n=-\infty}^{\infty} \delta(t-n T) x[n]\right] d \tau, \\
x[n]=\frac{1}{\sqrt{N}} \sum_{k=-\infty}^{\infty} \sum_{i=0}^{N-1} X_{i}^{(k)} e^{j(2 \pi i / N)(n-\nu-k L)} w_{r, s}[n-k L],
\end{gathered}
$$

with $\delta(t)$ the dirac impulse function, $T$ the sampling period, $w_{r, s}[n]$ a (rectangular, sampled) discrete time-domain window, $w_{r, s}[n]=1$ for $0 \leq n \leq L-1$ and zero elsewhere, and $v(t)$ an interpolation function.

The shape of the DMT spectrum will now be derived by construction, starting from a single symbol with only one active carrier at DC. This result will be extended to a succession of symbols with all carriers excited. After this, the influence of time-domain windowing will be investigated in Section 3.

Assume a single DMT symbol, having a duration $L=$ $N+v$ in which only the DC component is excited (e.g., with unit value), in other words,

$$
X_{i}^{(k)}= \begin{cases}1, & i=0, k=0 \\ 0, & \text { elsewhere }\end{cases}
$$

The corresponding discrete time-domain signal is a sequence of $\mathrm{L}$ identical pulses, which is equivalent to a multiplication of a rectangular window and an impulse train (Figure 2). A 


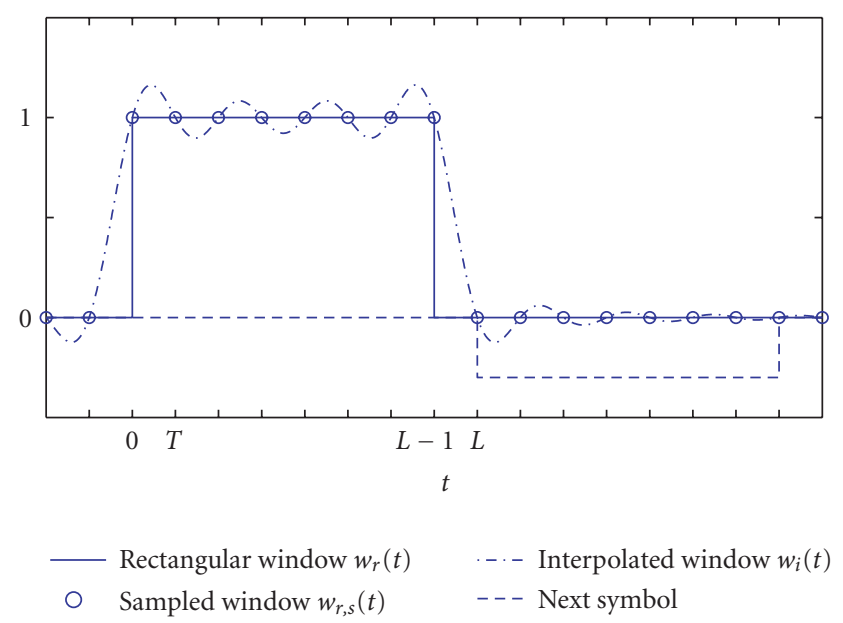

FIgURE 2: The first (DC only) symbol as a sampled rectangular window, and a possible next symbol.

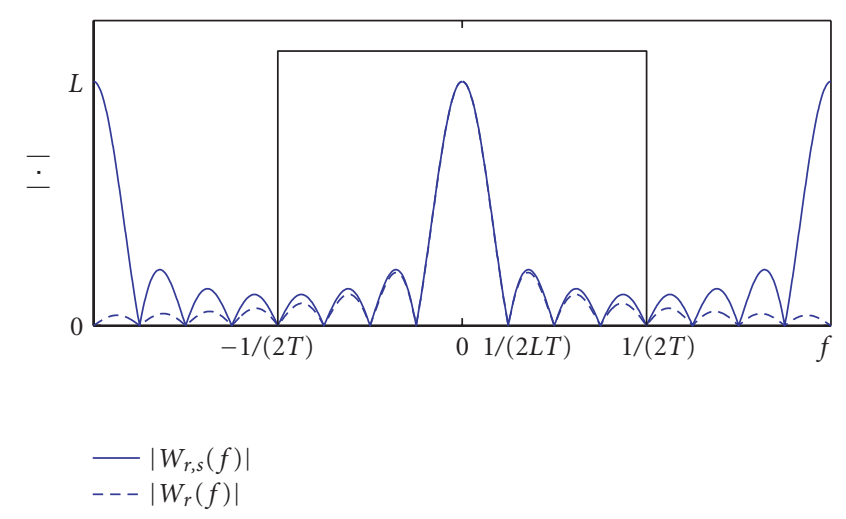

FIGURE 3: Spectrum of the continuous and sampled rectangular window.

rectangular window $w_{r}(t)$ extending from $t=0$ to $t=L$ has a modulated sinc as its Fourier transform

$$
W_{r}(f)=\frac{\sin (\pi L f)}{\pi f} \cdot \exp (-j \pi L f) .
$$

The multiplication of this $w_{r}(t)$ with a sequence of pulses with period $T$ results in the spectrum $W_{r}(f)$ being convolved with a pulse train with period $2 \pi / T$. The original sinc spectrum $W_{r}(f)$ and the convolved spectrum $W_{r, s}(f)$ are represented in Figure 3. Here, $W_{r, s}(f)$ is periodic with a period $1 / T$. Surprisingly, this can be expressed analytically as [16]

$$
W_{r, s}(f)=\frac{\sin (\pi L T f)}{\sin (\pi T f)} \exp (-j \pi L f) .
$$

In literature, $W_{r, s}(f)$ is sometimes approximated by a sinc. While this approximation is suitable for some applications, it leads to an underestimate of the (possible egress) energy in nonexcited frequency bands. More specifically, from (5), it is clear that this leads to a maximum error of $3.9 \mathrm{~dB}$ around $f= \pm 1 / 2 T$.

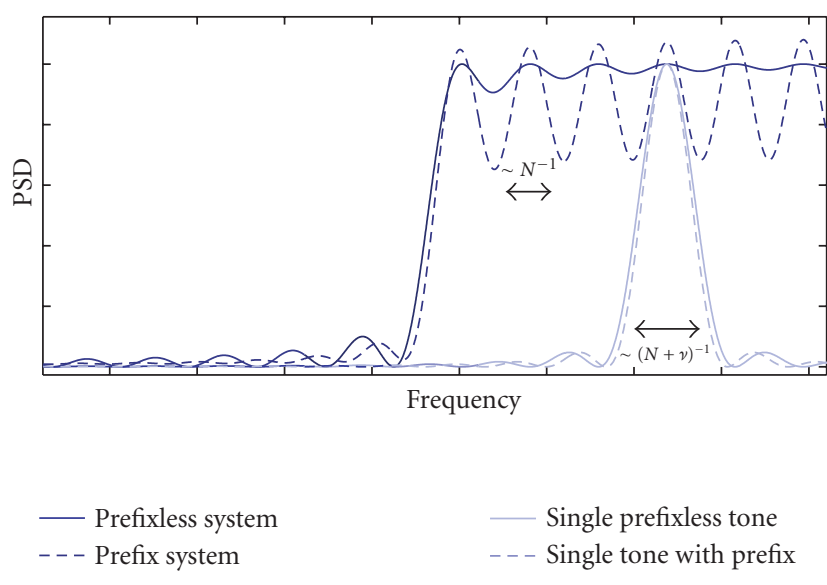

FIGURE 4: The cyclic prefix in DMT systems leads to a toothed spectrum exhibiting valleys in between the tones.

The final DA conversion consists of a lowpass filtering with $v(t)$, such that only the frequencies between $-1 / T$ and $1 / T$ are withheld. In the case of an ideal lowpass filter, this is equivalent to a time-domain interpolation with a sinc function, resulting in $w_{i}(t)$, as shown in Figure 2. Note that the continuous behaviour in between the sampled values is far from constant.

This result can now be extended to describe a succession of multiple symbols $(k=0,1, \ldots)$, with all tones $(i=$ $0,1, \ldots, N-1)$ excited. Assume that the $X_{i}^{(k)}$ have a variance $\mathcal{E}\left|X_{i}^{(k)}\right|^{2}=\sigma_{i}^{2}$, and are uncorrelated. The power spectral density (PSD) $S(f)$ of $s(t)$ can then be described as

$$
S(f)=\sum_{i=0}^{N-1} \sigma_{i}^{2}\left|W_{r, s}\left(f-\frac{i}{N T}\right) \cdot V(f)\right|^{2}
$$

with $V(f)$ the frequency characteristic of the interpolation filter $v(t)$ (an example of this is shown in Section 4).

Only in the case where the prefix is omitted $(\nu=0)$ and the variances $\sigma_{i}^{2}=\sigma^{2}$ are equal for all tones (except DC and the Nyquist frequency, having only $\sigma^{2} / 2$ ), this spectrum is more or less flat. In general, the CP results in a toothed spectrum. Indeed, because the symbols are lengthened by the CP, the PSD of the individual tones is narrowed compared to the intertone distance, such that "valleys" (or "teeth") appear in between the tone frequencies. This is demonstrated in Figure 4, where a detail of the spectrum of a prefixless DMT system $(\nu=0)$ is compared to a system using a prefix.

\section{TRANSMITTER WINDOWING}

Practical lowpass filters are not infinitely steep, such that some small signal components above the Nyquist frequency will remain. The out-of-band performance is then largely dependent on the quality of these filters (and possible clipping in further analog stages). On the other hand, the in-band 


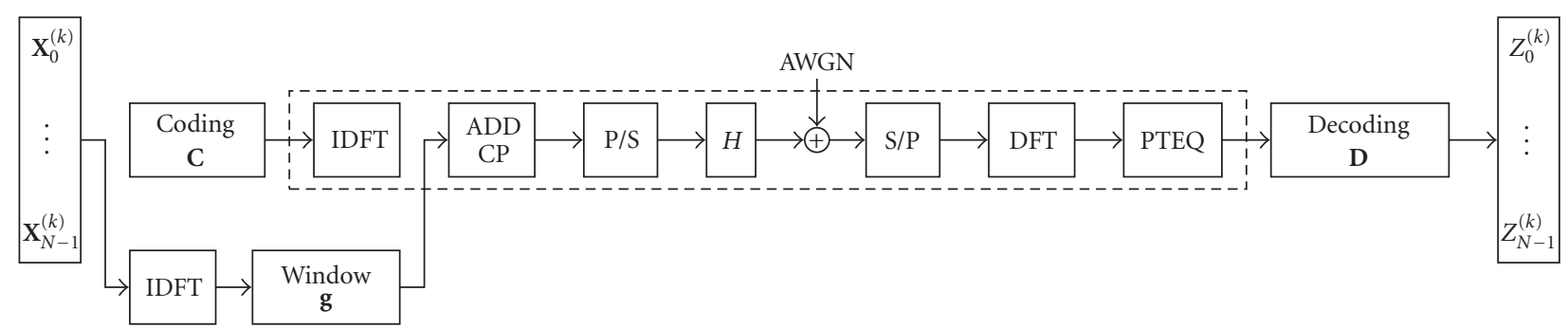

Figure 5: Transmitter windowing translates to symbol precoding.

transitions (e.g., for suppression of VDSL in the amateur radio bands) can only be sharpened by the application of a window function on the entire time-domain symbol. To achieve this, the rectangular window $w_{r, s}[n]$ is replaced by another one having faster decaying sidelobes. This new window

$$
\mathbf{w}=[w(0) \cdots w(L-1)]^{T}
$$

is applied at point $\alpha$ in Figure 1. In the next paragraph we impose constraints on $\mathbf{w}$ to construct a class of window functions that are easy to compensate for at the receiver.

\subsection{Derivation of the window structure}

To preserve the cyclic structure of the transmitted symbols, needed for an easy equalization, we impose the cyclic constraint

$$
w(n)=w(n+N), \quad n=0, \ldots, v-1 .
$$

As a result, instead of applying the window $\mathbf{w}$ at point $\alpha$ (Figure 1), one can also apply the window

$$
\begin{aligned}
\mathbf{g} & =[g(0) \cdots g(N-1)]^{T} \\
& =[w(v) \cdots w(N+v-1)]^{T}
\end{aligned}
$$

at point $\beta$. Let $\mathbf{G}$ be a diagonal matrix with $\mathbf{g}$ as its diagonal. After defining $\ell_{\mathrm{N}}$ the IDFT-matrix of size $N$, the vector of windowed samples $\mathbf{x}_{w}^{(k)}$ at point $\beta$ (before the application of the $\mathrm{CP}$ ) can be written as

$$
\mathbf{x}_{w}^{(k)}=\underbrace{\left[\begin{array}{cccc}
g(0) & 0 & \cdots & 0 \\
0 & g(1) & \ddots & 0 \\
\vdots & & \ddots & \vdots \\
0 & \cdots & 0 & g(n-1)
\end{array}\right]}_{\mathbf{G}} \ell_{\mathbf{N}} \cdot \mathbf{X}^{(k)} .
$$

As the product of a diagonal matrix and the IDFT-matrix is equal to the product of the IDFT-matrix and a circulant matrix, we can rewrite (10) as

$$
\mathbf{X}_{w}^{(k)}=\ell_{\mathbf{N}} \underbrace{\left[\begin{array}{cccc}
c(0) & c(1) & \cdots & c(N-1) \\
c(N-1) & c(0) & \ddots & c(N-2) \\
\ddots & & \ddots & \ddots \\
c(1) & \cdots & & c(0)
\end{array}\right]}_{\mathrm{C}} \cdot \mathbf{X}^{(k)} .
$$

The circulant matrix C (“C” for coding) is fully defined by its first row $\mathbf{c}^{T}$, with

$$
\mathbf{c}=[c(0) \cdots c(N-1)]^{T}=\boldsymbol{\ell}_{\mathbf{N}} \cdot \mathbf{g},
$$

that is, IDFT of $\mathbf{g}$. The transition from (10) to (11) is more than mathematical trickery. Looking at the DMT-scheme incorporating transmitter windowing of Figure 5, it becomes clear that the windowing operation in the time domain is equivalent to the multiplication of the subsymbol vector $\mathbf{X}^{(k)}$ with a (pre-)coding matrix $\mathbf{C}$. Compensating for the window at the receiver is now identical to a decoding in the frequency domain, which is done by multiplication with the decoding matrix $\mathbf{D}=\mathbf{C}^{-1}$ (" $\mathrm{D}$ " for decoding), leaving the rest of the signal path (equalization, etc.) unaltered. Thus, appealing windows should not only satisfy the constraint (8), but preferably also give rise to a sparse decoding matrix $\mathbf{D}$. We will now further investigate the nature of such windows.

Being the inverse of a circulant matrix, $\mathbf{D}$ is also circulant. We denote the first row of $\mathbf{D}$ as

$$
\mathbf{d}^{T}=[d(0) \cdots d(N-1)] .
$$

Define $\mathcal{F}_{\mathrm{N}}$ the DFT-matrix of size $N$, and

$$
\mathbf{f}=[f(0) \cdot f(N-1)]=\mathscr{F}_{\mathbf{N}} \cdot \mathbf{d} .
$$

It is now possible to associate to $\mathbf{D}$ a diagonal matrix $\mathbf{F}$, having on its diagonal the elements of $f$. The following relations now hold:

(i) $\mathbf{C}$ and $\mathbf{D}$ are circular, with $\mathbf{C}^{-1}=\mathbf{D}$, and have as a first row $\mathbf{c}^{T}$ and $\mathbf{d}^{T}$, respectively;

(ii) $\mathbf{G}$ and $\mathbf{F}$ are diagonal, with diagonals $\mathbf{g}$ and $\mathbf{f}$;

(iii) $\mathbf{c}=\ell_{\mathrm{N}} \cdot \mathrm{g}$;

(iv) $\mathbf{d}=\ell_{\mathbf{N}} \cdot \mathbf{f}$.

From this, we can conclude that $\mathbf{F}=\ell_{\mathbf{N}} \cdot \mathbf{D} \cdot \mathcal{F}_{\mathbf{N}}=\boldsymbol{\ell}_{\mathbf{N}} \cdot \mathbf{C}^{-1}$. $\tilde{F}_{\mathbf{N}}=\left(\ell_{\mathbf{N}} \cdot \mathbf{C} \cdot \mathcal{F}_{\mathbf{N}}\right)^{-1}=\mathbf{G}^{-1}$. In other words,

$$
g(n)=f(n)^{-1}, \quad n=0, \ldots, N-1 .
$$

Since $\mathbf{g}$ is real-valued, so is $\mathbf{f}$. Consequently, $\mathbf{d}$ is the IDFT of a real-valued vector. Because of the IDFT's symmetry properties, the first and middle elements of $\mathbf{d}$ are real-valued, and all other nonzero elements appear in complex conjugate pairs. 
We can now distinguish between three cases.

(i) A general $\mathbf{d}$ (nonsparse).

(ii) A maximally sparse $\mathbf{d}$ (with only three nonzero elements) is as follows:

$$
d(n)= \begin{cases}a, & n=0, \\ b \cdot e^{j \phi}, & n=l, \\ b \cdot e^{-j \phi}, & n=N-l, \\ 0, & n \notin\{0, l, N-l\},\end{cases}
$$

with

$$
\begin{aligned}
a, b & \text { real, } \\
\phi \quad \text { real } & \in\left[\begin{array}{ll}
-\pi & \pi
\end{array}\right], \\
l \text { integer } & \in\left[\begin{array}{lll}
1 & N-1
\end{array}\right],
\end{aligned}
$$

so that

$$
\mathbf{D}=\left[\begin{array}{ccccccc}
a & 0 & \cdots & 0 & b \cdot e^{j \phi} & 0 & \cdots \\
0 & \ddots & & & \ddots & \\
\vdots & & & & & \\
0 & & & & & \\
b \cdot e^{-j \phi} & & & & & \\
0 & \ddots & & & & \\
\vdots & & & & \ddots & \\
0 & & & & & a
\end{array}\right]
$$

is a sparse matrix. In practice, this means that $\mathbf{f}(\mathbf{f}=$ $\mathcal{F}_{\mathbf{N}} \cdot \mathbf{d}$ ) takes the form of a generalized raised cosine function. The different parameters influencing $\mathbf{f}$ are the pedestal height $a$, the frequency and amplitude of the sinusoidal part $l$ and $b$, and $\phi$ determining the position of the peak $(\mathrm{s})$.

(iii) Intermediate structures. Obviously, multiple complex pairs can be included (hence $5,7, \ldots$ nonzero elements in d), possibly leading to more powerful windows. A tradeoff should be made between the window quality and the complexity of the decoding.

\subsection{Determining the window parameters}

Returning to the original goal of egress reduction, we now need to choose $\mathbf{w}$ such that an improved sidelobe characteristic is obtained. For the rectangular window, the width of the mainlobe is equal to $\omega_{s}=2 \cdot \pi /(N+\nu)$. Note that this decreases with increasing CP length. As a general design criterion, we specify that the power outside the mainlobe $\omega_{s}=2 \cdot \pi /(N+\nu)$ should be as low as possible. Assuming that the total energy is kept constant, this is equivalent to maximizing the energy $\rho$ within the mainlobe [17], that is, maximizing

$$
\begin{gathered}
\rho=\int_{0}^{\omega_{s}}\left|W\left(e^{j \omega}\right)\right|^{2} \frac{d \omega}{\pi}, \\
\text { with } W(z)=\mathbf{w}^{T} \mathbf{e}(z), \\
\mathbf{e}(z)=\left[\begin{array}{llll}
1 & z & \cdots & z^{N+v-1}
\end{array}\right]^{T}
\end{gathered}
$$

under unit-energy constraint

$$
\mathbf{w}^{T} \cdot \mathbf{w}=1
$$

Equation (19) can be written as

$$
\begin{aligned}
\rho & =\mathbf{w}^{T}\left[\int_{0}^{\omega_{s}} \mathbf{e}^{*}\left(e^{j \omega}\right) \mathbf{e}\left(e^{j \omega}\right) \frac{d \omega}{\pi}\right] \mathbf{w} \\
& =\mathbf{w}^{T} \cdot \mathbf{Q} \cdot \mathbf{w}
\end{aligned}
$$

where $\mathbf{Q}$ has $(m, n)$ th entry

$$
\begin{aligned}
q_{\mathrm{mn}} & =\int_{0}^{\omega_{s}} \cos (m-n) \omega \frac{d \omega}{\pi}, \quad 0 \leq m, n \leq N+v-1 \\
& =\frac{\sin \left((m-n) \omega_{s}\right)}{(m-n) \pi}
\end{aligned}
$$

To enforce the cyclic structure (8), (24) is transformed into a problem in $\mathbf{g}$. After defining

$$
\mathbf{P}=\left[\begin{array}{cc}
\mathbf{O}_{\nu \times(N-v)} & \mathbf{I}_{\nu \times v} \\
\mathbf{I}_{N \times N} &
\end{array}\right],
$$

with $\mathbf{O}_{m \times n}$ and $\mathbf{I}_{m \times n}$ the all-zero and identity matrix of size $m \times n,(24)$ can be written as

$$
\rho=\mathbf{g}^{T} \cdot \mathbf{P}^{T} \cdot \mathbf{Q} \cdot \mathbf{P} \cdot \mathbf{g},
$$

and the unit-norm constraint becomes

$$
\mathbf{g} \cdot \mathbf{P}^{T} \cdot \mathbf{P} \cdot \mathbf{g}=1
$$

We can now again distinguish between three cases.

\section{(i) A general d (nonsparse)}

The maximization of (27) satisfying (28) can be rewritten as a generalized eigenvalue problem:

$$
\left(\mathbf{P}^{T} \mathbf{Q P}\right) \mathbf{g}=\lambda\left(\mathbf{P}^{T} \mathbf{P}\right) \mathbf{g}
$$

and the optimal vector $\mathbf{g}_{\text {opt }}$ is equal to the eigenvector corresponding to the largest eigenvalue of $\left(\mathbf{P}^{T} \mathbf{P}\right)^{-1} \mathbf{P}^{T} \mathbf{Q P}$. The optimal $\mathbf{w}_{\text {opt }}$ is now equal to

$$
\mathbf{w}_{\mathrm{opt}}=\mathbf{P} \cdot \mathbf{g}_{\mathrm{opt}} \cdot
$$

Note that $\mathbf{w}_{\text {opt }}$ is only dependent on the (chosen) width of the mainlobe.

\section{(ii) A maximally sparse d}

To obtain the optimal sparse decoding matrix $\mathrm{D}$, we have to determine the parameters $a, b, \phi$, and $l$ from (16) optimizing (27)-(28), with $\mathbf{f}=\mathcal{F}_{\mathbf{N}} \cdot \mathbf{d}$ and $g(n)=f(n)^{-1}, n=1, \ldots, N-$ 1 . We will use $l=1$, and $\phi$ such that $\mathbf{w}$ is symmetrical (i.e., $\phi=-v \pi / N)$. Due to the unit-energy constraint, only one of $a$ or $b$ can be chosen freely. This leads to a one-dimensional optimization problem in either $a$ or $b$. Because only three nonzero coefficients are present in $\mathbf{d}$, we denote this optimal (sparse) solution as $\mathbf{w}_{3 \text {,opt }}$ 


\section{(iii) Intermediate structures}

For the intermediate structures, multiple $(5,7, \ldots)$ nonzero elements are present in $\mathbf{d}$, leading to $\mathbf{w}_{5, \mathrm{opt}}, \mathbf{w}_{7, \mathrm{opt}}, \ldots$. These structures offer a tradeoff between egress reduction and computational complexity. The corresponding optimal windows are found using numerical optimization.

\subsection{Modification of the equalizer}

In the previous sections, it has been shown that the classical DMT structure can be modified to incorporate an encoding (C) and a decoding (D) to reduce the spectral leakage. The influence on the transmission itself was not mentioned so far and will now be investigated.

\section{(i) Approach-1: cascaded equalization and decoding}

In case the equalization of the received (encoded) symbols is perfect and in the absence of noise (i.e., if the dashed rectangle in Figure 5 is equal to a unity-matrix), it is obvious that the decoding will result in the original symbols. Because $\mathbf{D}=\mathbf{C}^{-1}$, it can be considered to be a decorrelator or zeroforcing equalizer (ZFE). Unfortunately in practical situations such a ZFE can enhance the noise. Moreover, it is not immediately clear how the equalizer itself (e.g., a PTEQ) should be designed in this case. Clearly this approach is not optimal.

\section{(ii) Approach-2: integrated per-tone equalization and decoding}

It turns out that the PTEQ can easily be modified to overcome both of the problems mentioned. To understand this, we first take a look at the structure of the original PTEQ (for details on its derivation, see [3]). An ordinary T-tap PTEQ for tone $i$ operates on received sample blocks of length $N+T-1$ and makes a linear combination of $i$ th output bin of a DFT and $T-1$ so-called difference terms which are common for all tones.

For the case of a maximally sparse $\mathbf{d}$ (16), the subsequent decoding (D) amounts to a linear combination of three of the PTEQ outputs. The result is now a linear combination of the difference terms and three output bins of the DFT.

The decoder and the PTEQ can now be easily combined by making one linear combination of the difference terms and three output bins of the DFT. This effectively increases the number of taps by two (for each tone), but solves both our problems.

(a) The PTEQ design criterion remains unchanged, only the number of inputs changes. Usually the PTEQ is designed to minimize the mean square error (MMSE) between the output and a known transmitted constellation point.

(b) the decoding is part of the equalizer and no longer represents a ZFE such that noise enhancement is avoided.

Obviously, selecting a $\mathbf{d}$ with additional nonzero elements will lead to an equalizer with an increased number of inputs, but it is based on the same principle. For the remainder of the article, we assume approach-2 is used.

The difference between approach- 1 and approach- 2 is illustrated in Figure 6.

\section{SIMULATION RESULTS}

\subsection{Influence on the egress}

Three windows are presented: the minimal window $\mathbf{w}_{3, \text { opt }}$ described by 3 nonzero coefficients in $\mathbf{d}$ (16), a slightly more complex window $\mathbf{w}_{5, \mathrm{opt}}$, for which $\mathbf{d}$ contains 5 nonzero coefficients, and the optimal window $\mathbf{w}_{\text {opt }}$ based on (29) and with nonsparse decoding.

The simulations have been done for a VDSL system. There are 2048 carriers $(N=4096)$, the prefix length is $C P=320$ (see [18, page 22]). The sampling frequency is $17664 \mathrm{kHz}$, the tone spacing is $4.3125 \mathrm{kHz}$.

In Figure 7, the shapes of the rectangular window, $\mathbf{w}_{3, \mathrm{opt}}$, $\mathbf{w}_{5, \text { opt }}$, and $\mathbf{w}_{\text {opt }}$ is shown. To illustrate the egress reduction, the spectra are compared for a VDSL scenario based on the power spectral density mask Pcab.P.M1 from [9]. The most important features are that the frequencies between $3000 \mathrm{kHz}$ and $5200 \mathrm{kHz}$ and above $7050 \mathrm{kHz}$ are reserved for upstream communications (see [18, page 17]), and that the power is lowered by $20 \mathrm{~dB}$ in the amateur radio bands, from $1810 \mathrm{kHz}$ to $2000 \mathrm{kHz}$, and from $7000 \mathrm{kHz}$ to $7100 \mathrm{kHz}$ (see [9, page 35]). The results are shown in Figures 8 and 9, showing a detail around the first amateur radio band. It is interesting to note that the spectrum is less toothed (the "valleys" in between the tones are less pronounced). Moreover, there is a significant egress reduction, especially around the band edge (about $5 \mathrm{~dB}$ ), achieved without adding any additional (redundant) cyclic extension. Obviously it would be possible to combine this method with such extensions.

Note that the sidelobe suppression of this technique in itself is not sufficient to allow the use of all tones up to the forbidden band. Other measures are necessary, such as leaving some tones unused close to the band edge. Note however that the number of unused (lost) tones will be lower than in case a rectangular window is used.

\subsection{Influence on the transmission}

As mentioned before, the PTEQ is usually designed according to an MMSE criterion. The exact solution to this problem requires a channel model and is very computationally demanding. Therefore, practical implementations generally use an adaptive scheme and a number of training symbols.

To make a fair comparison, however, we prefer the exact MMSE solution over an approach which relies on the convergence of the adaptive scheme. To reduce the simulation complexity, we then select an ADSL scenario. It can be expected that the obtained results are readily applicable to VDSL too.

More specifically, the simulations are done for an ADSL downstream scenario over a standard loop T1.601\#13, with $N=512, v=32$, and using tones 38 to 256 . The transmit power is $-40 \mathrm{dBm} / \mathrm{Hz}$ and additive white Gaussian noise of $-140 \mathrm{dBm} / \mathrm{Hz}$ was assumed. 


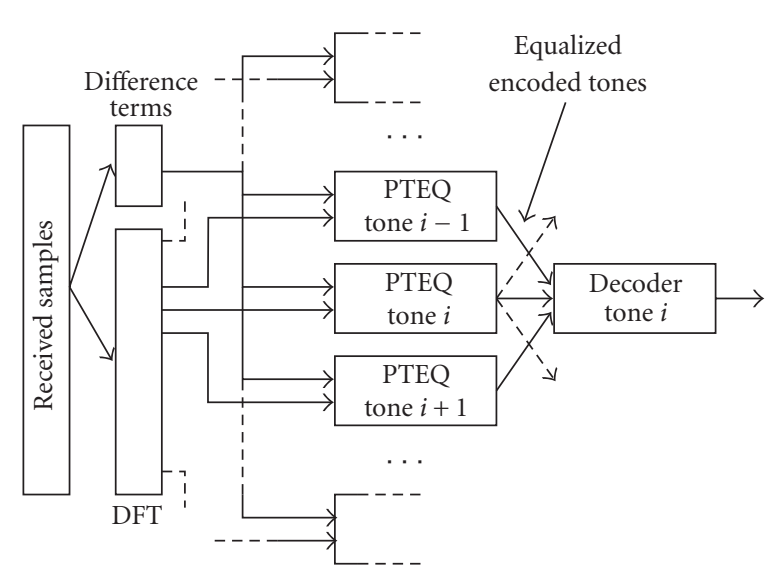

(a)

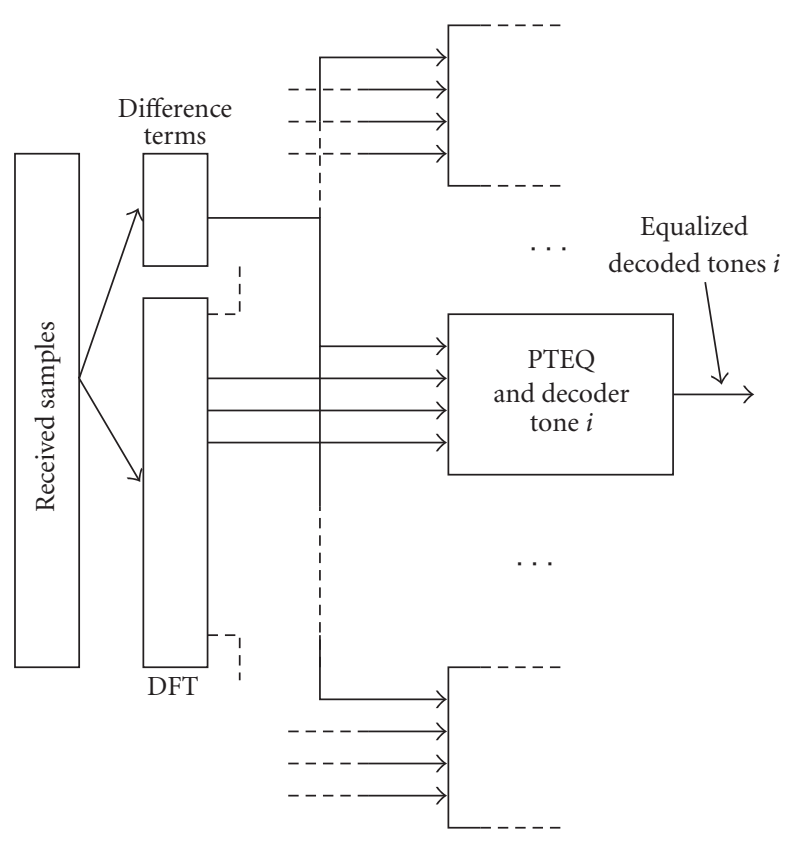

(b)

FIGURE 6: In approach-1 (left) the linear combiners (LC) of the PTEQ and the decoder are separated. In approach-2 (right) they are combined.

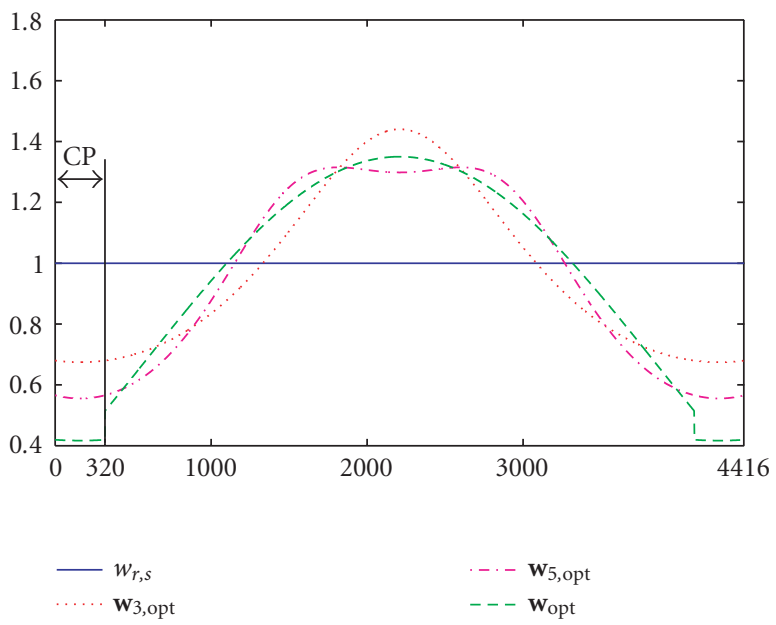

FIgURE 7: The shape of the rectangular window as well as $\mathbf{W}_{1, \mathrm{opt}}$, $\mathbf{W}_{2, \mathrm{opt}}$, and $\mathbf{W}_{\mathrm{opt}}$.

A system using a rectangular window at the transmitter and an ordinary PTEQ at the receiver is compared to a system using $\mathbf{W}_{3 \text {,opt }}$ (for ADSL dimensions) and approach-2 at the receiver. Note that this modified equalizer has the same number of taps as the ordinary PTEQ, implying that it uses 2 difference terms less, because these taps are assigned to the two additional DFT outputs.

The results are shown in Figure 10. For $T=3$, the performance of the proposed technique is significantly lower than that of the rectangular window combined with an ordinary

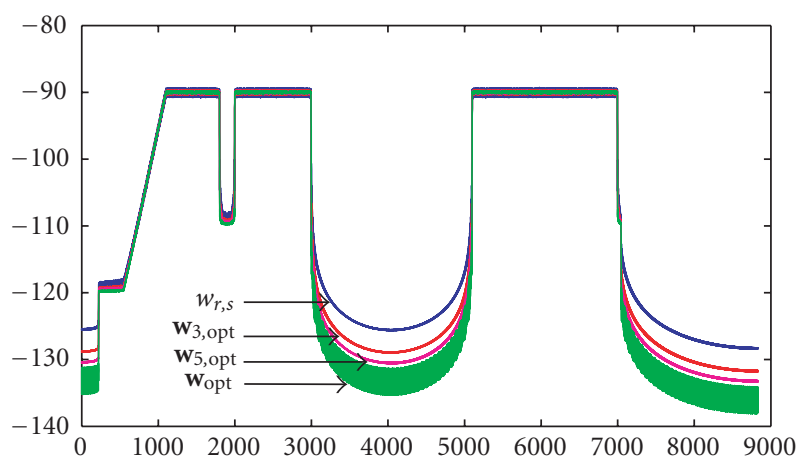

Figure 8: Spectrum of the rectangular window, $\mathbf{W}_{3, \mathrm{opt}}, \mathbf{W}_{5, \mathrm{opt}}$, and $\mathbf{W}_{\text {opt }}$.

PTEQ. This comes as no surprise because no taps are available for the difference terms, and the equalization is therfore poor. As the number of taps is increased, both techniques are very comparable.

\section{CONCLUSION AND FURTHER WORK}

A novel transmitter windowing technique for DMT has been proposed, which does not rely on an additional cyclic extension of the symbol. This inevitably introduces a distortion of the signal. For a special class of windows, this distortion can be described as a precoding operation for which the decoding at the receiver can be done easily. In the simplest case, the window function can be described as the pointwise inversion 


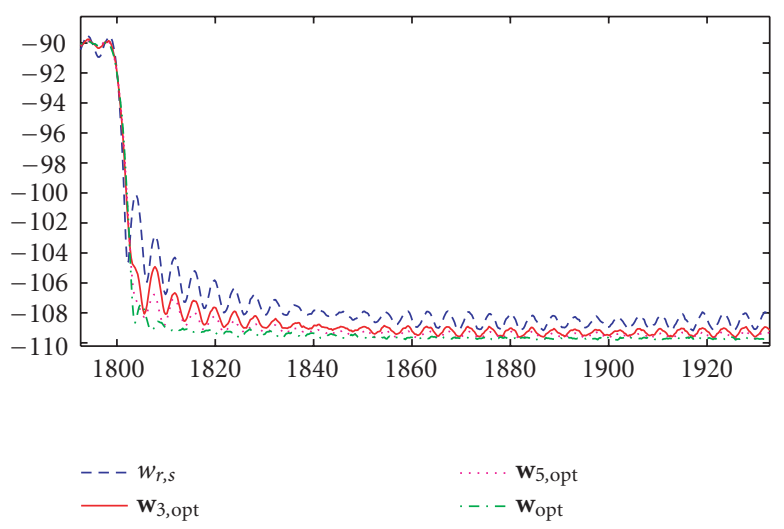

FIGURE 9: Spectrum of the rectangular window, $\mathbf{W}_{3 \text {,opt }}, \mathbf{W}_{5, \mathrm{opt}}$, and $\mathbf{W}_{\text {opt }}$ (detail of amateur radio band).

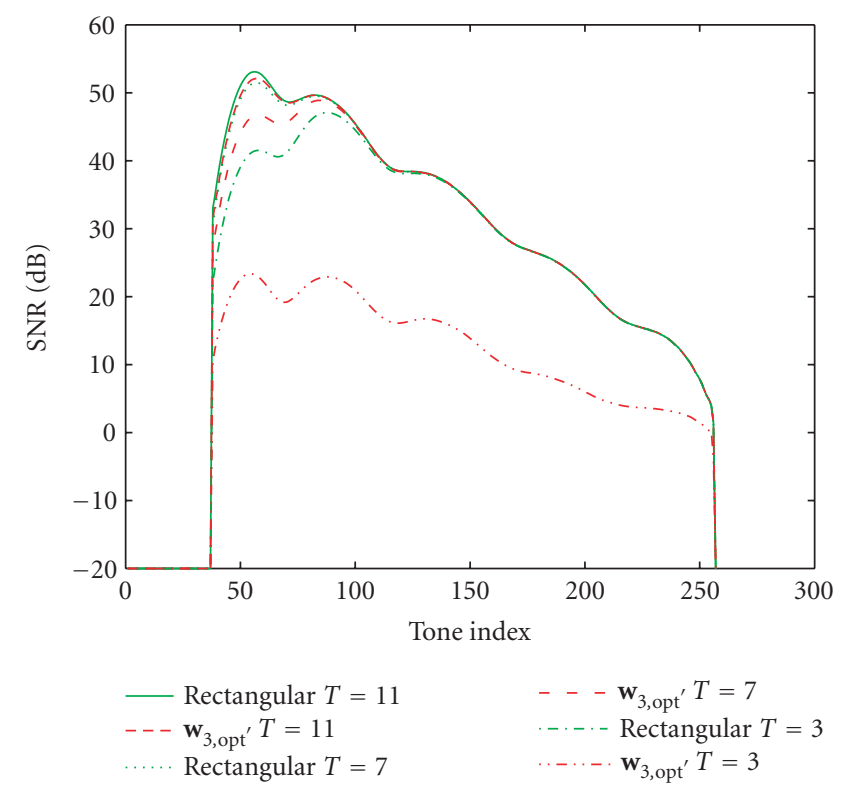

FIgURE 10: Comparison between the rectangular window using an ordinary PTEQ and the $\mathbf{W}_{3 \text {,opt }}$ window using approach-2.

of a raised cosine window. More complex windows can also be described, but the advantage of the easy decoding then gradually vanishes. Furthermore, formulas are provided to calculate the optimal window, and this is illustrated for the VDSL case.

The decoding at the receiver can be combined with a pertone equalizer in a very elegant way by taking into account additional DFT outputs. The effect on the transmission was illustrated for an ADSL scenario.

Future work will focus on a selective windowing of the tones in the vicinity of an unauthorized band, and the combination of the proposed technique with windowing in a cyclic extension of the symbol. Also the tradeoff between decoder complexity and egress should be further studied, as well as the interaction between the transmitter window and a channel equalizer using windowing at the receiver.

\section{ACKNOWLEDGMENTS}

This research work was carried out at the ESAT Laboratory of the Katholieke Universiteit Leuven, in the frame of Belgian Programme on Interuniversity Attraction Poles, initiated by the Belgian Federal Science Policy Office IUAP P5/22 ("Dynamical systems and control: computation, identification and modelling") and P5/11 ("Mobile multimedia communication systems and networks"), the Concerted Research Action GOA-AMBioRICS Research Project FWO no.G.0196.02 ("Design of efficient communication techniques for wireless time-dispersive multi-user MIMO systems”), CELTIC/IWT Project 040049: "BANITS" (Broadband Access Networks Integrated Telecommunications) and was partially sponsored by Alcatel Bell. The authors wish to thank the reviewers for their valuable comments and suggestions.

\section{REFERENCES}

[1] S. B. Weinstein and P. M. Ebert, "Data transmission by frequency-division multiplexing using the discrete fourier transform," IEEE Transactions on Communications, vol. 19, no. 5, pp. 628-634, 1971.

[2] A. Peled and A. Ruiz, "Frequency domain data transmission using reduced computational complexity algorithms," in Proceedings IEEE International Conference on Acoustics, Speech, and Signal Processing (ICASSP '80), vol. 5, pp. 964-967, Denver, Colo, USA, April 1980.

[3] K. Van Acker, G. Leus, M. Moonen, O. van de Wiel, and T. Pollet, "Per tone equalization for DMT-based systems," IEEE Transactions on Communications, vol. 49, no. 1, pp. 109-119, 2001.

[4] K. B. Song, S. T. Chung, G. Ginis, and J. M. Cioffi, "Dynamic spectrum management for next-generation DSL systems," IEEE Communications Magazine, vol. 40, no. 10, pp. 101-109, 2002.

[5] R. Stolle, "Electromagnetic coupling of twisted pair cables," IEEE Journal on Selected Areas in Communications, vol. 20, no. 5, pp. 883-892, 2002.

[6] A. J. Redfern, "Receiver window design for multicarrier communication systems," IEEE Journal on Selected Areas in Communications, vol. 20, no. 5, pp. 1029-1036, 2002.

[7] S. Kapoor and S. Nedic, "Interference suppression in DMT receivers using windowing," in Proceedings IEEE International Conference on Communications (ICC '00), vol. 2, pp. 778-782, New Orleans, La, USA, June 2000.

[8] G. Cuypers, G. Ysebaert, M. Moonen, and P. Vandaele, "Combining per tone equalization and windowing in DMT receivers," in Proceedings IEEE International Conference on Acoustics, Speech, and Signal Processing (ICASSP '02), vol. 3, pp. 2341-2344, Orlando, Fla, USA, May 2002.

[9] ETSI, "Transmission and Multiplexing (TM); Access transmission systems on metallic access cables; Very high speed Digital Subscriber Line (VDSL); part 1: Functional requirements," TS 101 270-1 V1.2.1 (1999-10), October 1999.

[10] K. W. Martin, "Small side-lobe filter design for multitone datacommunication applications," IEEE Transactions on Circuits and Systems-Part II: Analog and Digital Signal Processing, vol. 45, no. 8, pp. 1155-1161, 1998.

[11] G. Cherubini, E. Eleftheriou, and S. Ölçer, "Filtered multitone modulation for VDSL," in Proceedings IEEE Global Telecommunications Conference (GLOBECOM '99), vol. 2, pp. 11391144, Rio de Janeireo, Brazil, December 1999. 
[12] F. J. Harris, "On the use of windows for harmonic analysis with the discrete Fourier transform," Proceedings of the IEEE, vol. 66, no. 1, pp. 51-83, 1978.

[13] F. Sjöberg, R. Nilsson, M. Isaksson, P. Ödling, and P. O. Börjesson, "Asynchronous Zipper [subscriber line duplex method]," in Proceedings IEEE International Conference on Communications (ICC '99), vol. 1, pp. 231-235, Vancouver, BC, Canada, June 1999.

[14] Y.-P. Lin and S.-M. Phoong, "Window designs for DFT-based multicarrier systems," IEEE Transactions on Signal Processing, vol. 53, no. 3, pp. 1015-1024, 2005.

[15] H. Minn, C. Tellambura, and V. K. Bhargava, "On the peak factors of sampled and continuous signals," IEEE Communications Letters, vol. 5, no. 4, pp. 129-131, 2001.

[16] A. D. Poularikas, Handbook of Formulas and Tables for Signal Processing, CRC Press/IEEE, Boca Raton, Fla, USA, 1998.

[17] P. P. Vaidyanathan, Multirate Systems and Filter Banks, Prentice Hall, Englewood Cliffs, NJ, USA, 1st edition, 1993.

[18] ETSI, "Vdsl: Transceiver specification," TS 101 270-2 V1.1.1 (2001-02), 2001.

Gert Cuypers was born in Leuven, Belgium, in 1975. In 1998 he received the Master's degree in electrical engineering from the Katholieke Universiteit Leuven (KULeuven), Leuven, Belgium. Currently he is pursuing the Ph.D. degree at the Department of Electrical Engineering (ESAT), Leuven, Belgium, under the supervision of Marc Moonen. From 1999 to 2003, he was supported by the Flemish Institute for Scientific and

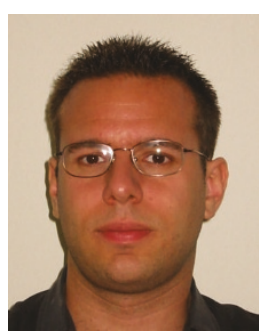
Technological Research in Industry (IWT). At the moment he teaches at the Leuven Engineering School (Groep T), Leuven, Belgium. His interests are in digital communications and RF technology. His amateur radio call sign is ON4DSP.

Koen Vanbleu was born in Bonheiden, Belgium, in 1976. He received the Master's and Ph.D. degrees in electrical engineering from the Katholieke Universiteit Leuven (KULeuven), Leuven, Belgium, in 1999 and 2004, respectively. From 1999 to 2003, he was supported by the Fonds voor Wetenschappelijk Onderzoek (FWO) Vlaanderen. At the moment he works for Broadcom (Belgium).

Geert Ysebaert was born in Leuven, Belgium, in 1976. He received the Master's and the Ph.D. degrees in electrical engineering from the Katholieke Universiteit Leuven (KULeuven), Leuven, Belgium, in 1999 and 2004, respectively. From 1999 to 2003, he was supported by the Flemish Institute for Scientific and Technological Research in Industry (IWT). In September 2004, he joined

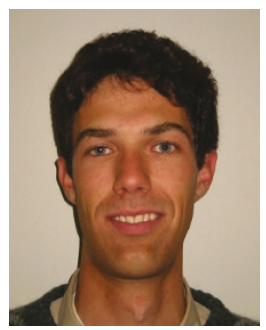
the DSL Experts Team at Alcatel Bell, where he is involved in dynamic spectrum management (DSM), single ended line testing (SELT), and quality of service (QoS) for DSL. He is married to Ilse and has a baby named Roan.

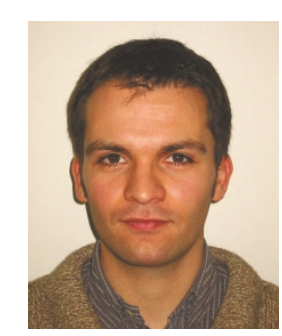

Marc Moonen received the Electrical Engineering degree and the Ph.D. degree in applied sciences from Katholieke Universiteit Leuven, Leuven, Belgium, in 1986 and 1990, respectively. Since 2004 he is a Full Professor at the Electrical Engineering Department of Katholieke Universiteit Leuven, where he is currently heading a research team of 16 Ph.D. candidates and postdocs, working in

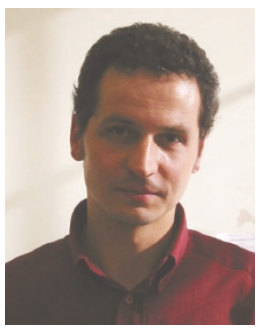
the area of signal processing for digital communications, wireless communications, DSL, and audio signal processing. He received the 1994 K.U. Leuven Research Council Award, the 1997 Alcatel Bell (Belgium) Award (with Piet Vandaele), the 2004 Alcatel Bell (Belgium) Award (with Raphael Cendrillon), and was a 1997 "Laureate of the Belgium Royal Academy of Science". He was the Chairman of the IEEE Benelux Signal Processing Chapter (1998-2002), and is currently a EURASIP AdCom Member (European Association for Signal, Speech and Image Processing, from 2000 till now). He has been a Member of the Editorial Board of "IEEE Transactions on Circuits and Systems II" (2002-2003). He is currently the Editor-in-Chief for the "EURASIP Journal on Applied Signal Processing" (from 2003 till now), and a Member of the Editorial Board of "Integration, the VLSI Journal", "EURASIP Journal on Wireless Communications and Networking", and "IEEE Signal Processing Magazine”.

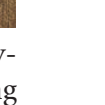

